\pm 0.28 ischaemic/control limb ratio; $n=8, p<0.05)$ CB-ECFCs into mouse ischaemic hindlimbs inhibited and promoted revascularisation whilst regulating host eNOS-associated angiogenic signalling. Together, these findings indicate a key role for NOX4 in CB-ECFCs, highlighting its potential as a target for enhancing their reparative function through therapeutic priming to support creation of a pro-reparative microenvironment and promotion of effective post ischaemic revascularisation.

\section{INVESTIGATING THE COUNTER REGULATORY RENIN ANGIOTENSIN SYSTEM AXIS IN THE STROKE PRONE SPONTANEOUSLY HYPERTENSIVE RAT IN ISCHAEMIC STROKE}

${ }^{1}$ Aisling McFall, ${ }^{2}$ Lorena Zentilin, ${ }^{2}$ Mauro Giacca, ${ }^{1}$ Stuart A Nicklin, ${ }^{1}$ Lorraine M Work. ${ }^{1}$ Institute of Cardiovascular and Medical Sciences, University of Glasgow, UK; ${ }^{2}$ International Centre for Genetic Engineering and Biotechnology, Trieste, Italy

\subsection{6/heartjnl-2018-SCF.9}

Several studies have assessed the potential of targeting the renin angiotensin system (RAS) with therapeutics for ischaemic stroke. The counter regulatory RAS peptide, angiotensin-(1-9) has been shown to act via the angiotensin II type 2 receptor $\left(\mathrm{AT}_{2} \mathrm{R}\right)$ to oppose detrimental effects of RAS dysregulation. We hypothesise that Ang-(1-9) may have a beneficial effect on stroke outcome in the spontaneously hypertensive stroke prone rat (SHRSP). Initial qPCR experiments have assessed temporal changes in RAS gene expression (angiotensin converting enzyme 2; ACE2, $\mathrm{AT}_{2} \mathrm{R}$; AGTR2, Mas receptor; Mas) following $35 \mathrm{~min}$ transient middle cerebral artery occlusion (tMCAO) followed by varying reperfusion times: no reperfusion $(n=4), 2$ hour $(n=4)$ and 24 hour $(n=4)$, compared to sham surgery $(n=7)$.

In infarcted tissue, there was a significant 10- and 11-fold reduction in ACE2 and Mas expression respectively, 24 hour post $\mathrm{tMCAO}$ vs sham $\left(\mathrm{RQ}+\mathrm{RQ}_{\max }\right.$ : ACE2: sham $1.0+0.2$; 24 hour post tMCAO $0.1+0.01, \mathrm{p}<0.01$, Mas: sham $1.0+0.2$; 24 hour post tMCAO $0.09+0.03 \mathrm{p}<0.01)$. However, in the same tissue, AGTR2 showed a 4-fold increase in expression after 35 min occlusion vs sham $\left(\mathrm{RQ}+\mathrm{RQ}_{\max }\right.$ : sham $1.0+0.3$; 35 min MCAO 4.2+0.2, $\mathrm{p}<0.05)$. Additionally, in the subcortical remainder tissue, ACE2 and AGTR2 expression decreased by 2.5 - and 5-fold respectively 24 hour post tMCAO (RQ+RQ $\mathrm{Rax}_{\text {ax }}$ ACE2: sham $1.0+0.1 ; 24$ hour post tMCAO $0.4+0.1, \mathrm{p}<0.05$, AGTR2: sham $1.0+0.4 ; 24$ hour post tMCAO $0.2+0.1 \mathrm{p}<0.05)$.

These results demonstrate altered counter regulatory RAS gene expression in the ipsilateral hemisphere in the 24 hours following tMCAO in SHRSP. Additional experiments have demonstrated successful transduction of a control reporter gene-expressing, adeno-associated virus serotype 9 (AAV9) expressing enhanced green fluorescent protein (eGFP) (AAV9eGFP) in the SHRSP brain via stereotactic delivery after both 4 and 7 days. Future studies will assess the therapeutic potential of Ang-(1-9) in tMCAO induced experimental stroke in SHRSP by delivering Ang-(1-9) via stereotactic delivery of an AAV9 vector.

\section{ENDOGENOUS AND EXOGENOUS LOADING OF EXTRACELLULAR VESICLES FOR THERAPEUTIC DELIVERY OF RENIN-ANGIOTENSIN SYSTEM PEPTIDES IN CARDIOMYOCYTE HYPERTROPHY}

LS Downie, LM Work, SA Nicklin. Institute of Cardiovascular and Medical Sciences, University of Glasgow

10.1136/heartjnl-2018-SCF.10

Introduction The RAS peptide angiotensin II (AngII) mediates cardiac hypertrophy. The counter-regulatory RAS axis peptide Angiotensin 1-7 [Ang-(1-7)] inhibits cardiomyocyte hypertrophy. EVs were purified from cardiomyocytes \pm treatment with AngII or Ang-(1-7) to assess cardiomyocyte hypertrophy. EVs were loaded with Ang-(1-7) via electroporation for therapeutic delivery.

Methods H9c2 cardiomyocytes were untreated (control) or treated with AngII or Ang-(1-7). EVs were isolated from conditioned media by differential ultracentrifugation, characterised by BCA, western immunoblot, Nanosight and TEM and incubated with recipient cardiomyocytes. Next, cells were stained with F-Phalloidin actin and area measured. Gene expression of hypertrophy marker brain natriuretic peptide (BNP) was assessed by qRT-PCR. Control EVs were electroporated in the presence of Ang-(1-7) and levels determined by ELISA.

Results H9c2 cardiomyocyte-derived EV size was 101.0 $\pm 2.4 \mathrm{~nm}$ and EV markers CD63 and TSG-101 were consistently detected. EVs from AngII treated cardiomyocytes significantly increased recipient cardiomyocyte area compared to control EVs [control: 3291.1 $\pm 90.1 \mu^{2}$ vs AngII:5252.3 $\left.\pm 125.4 \mu^{2} ; \quad p<0.001\right]$ and significantly increased BNP expression $[p<0.017]$. EVs isolated from Ang-(1-7) treated $\mathrm{H} 9 \mathrm{c} 2$ cardiomyocytes significantly reduced AngII induced hypertrophy in recipient cardiomyocytes [AngII +Control EVs:5566.3 $\pm 139.0 \mu \mathrm{m}^{2}$ vs AngII + Ang-(1-7) EVs:4212.7 $\left.\pm 132.1 \mu \mathrm{m}^{2} ; \mathrm{p}<0.01\right]$. Electroporation loaded EVs with Ang(1-7) [naïve EVs:0.0 pg/mL vs Ang-(1-7) EVs:342.3 $\pm 9.1 \mathrm{pg} /$ $\mathrm{mL} ; \mathrm{p}<0.001]$. Ang-(1-7) loaded EVs significantly reduced AngII induced hypertrophy in recipient cardiomyocytes [Naive EVs:4641.2 $\pm 35.3 \mu \mathrm{m}^{2}$ vs Ang-(1-7) EVs:2758.4 $\pm 20.1 \mu \mathrm{m}^{2}$; $\mathrm{p}<0.001]$.

Conclusion EVs isolated from AngII treated H9c2 cardiomyocytes stimulate recipient cardiomyocyte hypertrophy. EVs isolated from Ang-(1-7) treated cardiomyocytes inhibit hypertrophy. Furthermore, EVs exogenously loaded with Ang(1-7) inhibit cardiomyocyte hypertrophy. These findings have implications for understanding the role of the RAS and EV function in cardiomyocytes.

\section{Poster Presentations}

\section{A SURVEY EVALUATING HEALTHCARE PROFESSIONALS' KNOWLEDGE AND PERCEPTIONS OF ELECTRONIC CIGARETTES}

Dickson Dewantoro, Danièle MI Kerr, Christian Delles. Institute of Cardiovascular and Medical Sciences, University of Glasgow, UK

10.1136/heartjnl-2018-SCF.11 